\title{
Lentivirus-mediated overexpression of CD97/ADGRE5 reverses dysregulated high glucose-induced endothelial cell migration
}

\author{
WENTING ZHAO, ZHEN WANG, ZEWEI SUN, SHUAI WANG, MINGJIE WU and LIANGRONG ZHENG \\ Department of Cardiology, The First Affiliated Hospital, School of Medicine, \\ Zhejiang University, Hangzhou, Zhejiang 310003, P.R. China
}

Received January 6, 2016; Accepted December 21, 2016

DOI: $10.3892 / \mathrm{mmr} .2017 .6417$

\begin{abstract}
Elevated blood glucose levels contribute to a series of complications in patients with diabetes mellitus, including chronic ulcers and accelerated atherosclerosis. Dysregulated endothelial migration induced by high glucose is important in vascular-associated complications. In the present study, cluster of differentiation (CD)97/adhesion G protein-coupled receptor 5 (ADGRE5), a member of the G protein-coupled receptor protein family, stimulated angiogenesis was investigated to determine its role in cell models of diabetes mellitus using lentivirus-mediated overexpression and siRNA transfection. The results revealed reduced expression in high glucose-treated human umbilical vein endothelial cells and in the endothelium of diabetic mice. Among the three CD97 isoforms, the majority of the expression of CD97 (EGF1,2,5) in the endothelial cells was regulated by high levels of glucose. Using stable lentivirus-mediated transfection and clustered regularly interspaced short palindromic repeats (CRISPR)/CRISPR-associated protein 9 (Cas9) technology, the present study constructed CD97-overexpression and CD97-knockout endothelial cell lines. Migration assays showed that the lentivirus-mediated overexpression of CD97/ADGRE5 improved the inhibition of high glucose-induced endothelial cell migration. In addition, using cytoskeleton staining, it was found that CD97 promoted membrane ruffling and lamellipodia formation. Cell division cycle 42, a small GTP-binding protein, and its downstream factor, actin-related protein 2, were involved in CD97-induced actin reorganization in endothelial cells. Additionally, the use of transcription factor filter plate assays revealed that the nuclear translocation of signal
\end{abstract}

Correspondence to: Dr Liangrong Zheng, Department of Cardiology, The First Affiliated Hospital, School of Medicine, Zhejiang University, 79 Qingchun Road, Hangzhou, Zhejiang 310003, P.R. China

E-mail: zlrylnn@126.com

Key words: cluster of differentiation 97, high glucose, migration, cell division cycle 42, actin-related protein 2 transducer and activator of transcription 1 stimulated by high glucose contributed to the inhibited transcription of CD97. In conclusion, the present study established that the overexpression of CD97 improved high glucose-induced dysfunction of endothelial cell migration. These findings provide insight to assist in identifying therapeutic targets with potential to ameliorate certain vascular complications of diabetes.

\section{Introduction}

Endothelial dysfunction associated with type 2 diabetes mellitus and insulin resistance (1) is involved in multiple complications of diabetes. Numerous studies have suggested that endothelial cell dysfunction increases macromolecule permeability $(2,3)$, causes abnormal vasodilation/vasoconstriction (4-6) and results in procoagulant activation (7).

Endothelial migration contributes to vascular repair and is inhibited under conditions of high glucose (8). Furthermore, previous data have shown that cluster of differentiation (CD)97, a surface molecule abundantly expressed in endothelial cells, can stimulate migration, invasion and angiogenesis (9). Therefore, the present study hypothesized that CD97 may act to promote endothelial cell migration under high glucose treatment conditions.

CD97 is encoded by the adhesion G protein-coupled receptor 5 (ADGRE5) gene (10) and is a member of the epidermal growth factor (EGF)-seven transmembrane family, which belongs to the adhesion family of $\mathrm{G}$ protein-coupled receptors (GPCRs) (11-13). CD97 is widely expressed on the surface of lymphoid cells, macrophages, smooth muscle cells and several types of tumor cell (14-19). A previous study also found that CD97 enhances cell invasion via Ras homolog (RHO) and extracellular signal-regulated kinase activation by associating with lysophosphatidic acid receptor 1 in prostate cancer cells (19).

In the present study, a lentivirus-mediated endothelial cell line overexpressing CD97 was constructed and the effects of CD97 on cell migration were investigated. It was found that CD97 ameliorated the inhibition of high glucose-induced endothelial cell migration. In addition, the molecular mechanism whereby high levels of glucose regulate the expression of CD97 was also characterized in detail. 


\section{Materials and methods}

Cell culture. The human umbilical vein endothelial cell (HUVEC) line was purchased from American Type Culture Collection (Manassas, VA, USA). The cells were cultured in an incubator at $37^{\circ} \mathrm{C}, 5 \% \mathrm{CO}_{2}$ in complete medium containing $10 \%$ FBS (Sigma-Aldrich; Merck Millipore, Darmstadt, Germany) and Dulbecco's modified Eagle's medium (DMEM; $5.5 \mathrm{mM}$; Gibco; Thermo Fisher Scientific, Inc., Waltham, MA, USA). Prior to exposure to glucose at concentrations of 5.5, 10 or $33 \mathrm{mM}$ for $24 \mathrm{~h}, 1 \times 10^{5}$ cells were starved of FBS for $12 \mathrm{~h}$ at $37^{\circ} \mathrm{C}$ in the incubator. A $5.5 \mathrm{mM}$ concentration of glucose was used as a control.

Small interfering (si)RNA and plasmid transfection. Transfection of the cells was performed using Polyplus transfection reagent (jetPPRIME; Polyplus Transfection, Illkirch, France). In brief, $1 \mu \mathrm{l}$ of siRNA (50 $\mu \mathrm{M}$; Ruibo Biotechnology Co., Ltd., Guangzhou, China) or $2.5 \mu \mathrm{g}$ plasmid cDNA (ViGene Biosciences, Inc., Shandong, China) was added to $200 \mu \mathrm{l}$ of jetPRIME buffer. Following mixing with $4 \mu \mathrm{l}$ of jetPRIME, the solution was vortexed for $10 \mathrm{sec}$. Following incubation for $10 \mathrm{~min}$ at room temperature, the mixture was added into one well of a 6 -well plate with $1 \times 10^{6}$ cells cultured in $1 \mathrm{ml}$ complete medium. Following culture for an additional $24 \mathrm{~h}$, the cells were harvested and used in subsequent assays.

Western blot analysis. The cells were lysed on ice in RIPA lysis buffer (Beyotime Institute of Biotechnology, Shanghai, China), which included a cocktail of protease inhibitors (Cell Signaling Technology, Inc., Danvers, MA, USA) and protein levels was determined by bicinchoninic acid assay method. Subsequently, $40 \mu \mathrm{g}$ of total protein was loaded onto $10 \%$ SDS-PAGE gels, electrophoresed and transferred onto PVDF membranes (EMD Millipore, Billerica, MA, USA). Following blocking using 5\% non-fat milk (Nestlé, Vevey, Switzerland), antibody incubation and immunoblotting using ECL (Kangwei, Beijing China) were used to detect fluorescence. The following primary antibodies were used: anti-human CD97 (cat. no. ab108368; 1:1,000; Abcam, Cambridge, MA, USA), anti-human rhodopsin (RHO; cat. no. ab5417; 1:1,000; Abcam), anti-human Ras-related C3 botulinum toxin substrate 1 (RAC; cat. no. ab33186; 1:1,000; Abcam), anti-human cell division cycle 42 (CDC42; cat. no. ab155940; 1:1,000; Abcam), anti-human actin-related protein 2 (ARP2; cat. no. ab47654; 1:1,000; Abcam), anti-human signal transducer and activator of transcription 1 (STAT1; cat. no. ab3987; 1:1,000; Abcam) and anti-human GAPDH (cat. no. CW0101M; 1:2,000; Nuoyang, Hangzhou, China). All primary antibodies were incubated at $4^{\circ} \mathrm{C}$ overnight. The relevant secondary antibody was goat anti-rabbit antibody (cat. no. A0208; 1:5,000; Beyotime Institute of Biotechnology) incubated at room temperature for $1 \mathrm{~h}$.

Flow cytometry. Following treatment, the cells were washed twice with PBS and then incubated with FITC-conjugated anti-CD97 (BD Biosciences, Franklin Lakes, NJ, USA). The subsequent analysis of the expression of CD97 was performed using a flow cytometer (BD Biosciences).
Reverse transcription-polymerase chain reaction ( $R T-P C R)$ analysis. Total RNA was extracted from the endothelial cells using an RNAsimple total RNA kit (Tiangen Biotech Co., Ltd., Beijing, China). First-strand cDNA was synthesized using a Primescript RT reagent kit (Takara Bio, Inc., Otsu, Japan). A total of $5 \mathrm{ng}$ cDNA of each sample was subjected to PCR reactions consisting of 40 cycles of $95^{\circ} \mathrm{C}$ for $10 \mathrm{sec}, 68^{\circ} \mathrm{C}$ for $30 \mathrm{sec}$ and $72^{\circ} \mathrm{C}$ for $30 \mathrm{sec}$ using SYBR-Green Premix Ex Taq (Takara Bio, Inc., Otsu, Japan) and detected by ABI PRISM 7500 Sequence Detection System (Thermo Fisher Scientific, Inc.). The relative expression level results were analyzed using the $2^{-\Delta \Delta C q}$ method (20). Finally, the PCR products were examined using DNA agarose gel electrophoresis. The following primers were used for RT-PCR analysis: CD97, forward 5'-ACTCTGCCGGGAGCTGAAAC-3' and reverse 5'-TGGATGGTGACCTCGGCTGA-3'; 18S, forward 5'-CCG CACTTGATACGGTTCCT-3' and reverse 5'-CCAGGCTGA TCTATCCCACTG-3'.

Wound healing assay. Endothelial cells were seeded at density $1 \times 10^{5}$ and cultured in 6-well plates to $80-90 \%$ confluence and were serum-starved for $24 \mathrm{~h}$. Two scratches were then introduced to the cell layer in each well using a 100-1,000 $\mu 1$ tip. Following washing twice with PBS, the cells were incubated in DMEM with glucose (33 mM), and $5.5 \mathrm{mM}$ glucose DMEM treatment was used as a control. Images of the same regions were captured at 0 and $24 \mathrm{~h}$ following stimulation with light microscope (Stemi 2000; Zeiss GmbH, Jena, Germany); the paired images were analyzed.

Chromatin immunoprecipitation (ChIP). ChIP assays were performed according to the manufacturer's protocol using a kit from Cell Signaling Technology, Inc. In brief, for each group, $1 \times 10^{7}$ cells were fixed with $1 \%$ formaldehyde (Aladdin Industrial, Inc., Nashville, TN, USA). Subsequently, chromatin DNA was sheared using micrococcal nuclease (Cell Signaling Technology, Inc.) to yield DNA fragments ranging between 300 and $900 \mathrm{bp}$. Following preclearance with $10 \mu \mathrm{l}$ protein $\mathrm{A} / \mathrm{G}$ agarose beads, the samples were incubated with rabbit anti-STAT1 monoclonal antibody ( $2 \mu \mathrm{g}$; cat. no. ab3987; 1:100; Abcam) or control rabbit IgG antibody ( $2 \mu \mathrm{g}$; cat. no. 2729; 1:100; Cell Signaling Technology, Inc.). The samples were then immunoprecipitated by incubation with $30 \mu 1$ protein $\mathrm{A} / \mathrm{G}$ agarose beads, and complexes were reverse cross-linked by protease $\mathrm{K}$ and $\mathrm{NaCl}$ (5 M) treatment. Finally, DNA was purified using a DNA purification kit (Cell Signaling Technology, Inc.). The content of the purified DNA was assessed using RT-quantitative PCR analysis as aforementioned. The following primers were used to amplify the STAT1 binding element in the promoter of the CD97 gene: Forward 5'-TAG CGCTAAGACACAGTTGGACC-3' and reverse 5'-ACTCGC CAGTTGCAACAGTTC-3'.

Generation of the CD97-Cas9 endothelial cell line. Toknockout CD97 in an endothelial cell line, experiments were performed according to a previously published protocol (21). In brief, a custom designed gRNA for CD97 was cloned into the Pep-ko (Pep-330x) plasmid. Then the plasmid was transfected into endothelial cells at a density $3 \times 10^{5}$ using Polyplus transfection reagent (JetPRIME; Polyplus Transfection) at $37^{\circ} \mathrm{C}$ for $24 \mathrm{~h}$, 
the endothelial cells were filtered using puromycin $(2 \mu \mathrm{g} / \mathrm{ml}$; Sigma-Aldrich; Merck Millipore). The surviving cells were seeded into a 96-well plate and cultured into monoclonal cell lines for further assessment of the expression of CD97. The CD97 gRNA sequences were as follows: Forward 5'-accgTC CGGTGGACGAGGCGGCGG-3' and reverse 5'-accgCGGCC GACCACCACCGCTTC-3'.

Construction of stable CD97-expressing endothelial cells by lentivirus transfection. A customized CD97-overexpression lentivirus vector was obtained from ViGene Biosciences, Inc. Endothelial cells were transfected with the CD97 lentivirus and screened/selected using puromycin $(2 \mu \mathrm{g} / \mathrm{ml}$; Sigma-Aldrich; Merck Millipore). The surviving cells were cultured into multiple monoclonal cell lines and were assessed for the expression of CD97 using western blot analysis.

Immunofluorescence staining. Endothelial cells were seeded at a density of $1 \times 10^{5}$ in a cell culture dish (Nest Scientific, Rahway, NJ, USA). Following treatment with glucose (control, $5.5 \mathrm{mM}$; high, $33 \mathrm{mM}$ ) for $24 \mathrm{~h}$, the cells were fixed using $1 \%$ formaldehyde and incubated with FITC-phalloidin (Thermo Fisher Scientific, Inc.). Finally, the cells were observed using a Zeiss Confocal Imaging system (Zeiss $\mathrm{GmbH}$ ).

Animal model. A total of 20 male C57BL/6 J mice (age, 4 weeks; weight, $20 \pm 4 \mathrm{~g}$, maintained at $20^{\circ} \mathrm{C}$, normal lighting) were purchased from the Shanghai Institute for Biological Sciences (Shanghai, China). Diabetes was induced in these mice by intraperitoneal injection of STZ $(70 \mathrm{mg} / \mathrm{kg}$; Sigma-Aldrich; Merck Millipore), and the mice were continually fed a high-fat diet for 3 months. Mice with glucose levels $>16.4 \mathrm{mM}$ were considered a successful diabetic mouse model. Three months later, mice were sacrificed by cervical dislocation. The aortic endothelium was harvested and subjected to analysis of the expression of CD97 using immunohistochemistry (IHC).

IHC. Following deparaffinization, hydration and blocking, the paraffin-embedded tissue transverse sections (thickness, $5 \mu \mathrm{m}$ ) were incubated with primary antibodies (CD97; cat. no. ab108368, 1:200; Abcam) for $2 \mathrm{~h}$ at $37^{\circ} \mathrm{C}$. Following incubation, the tissue sections were washed with PBS for $15 \mathrm{~min}$, followed by incubation with anti-digoxigenin-conjugated secondary antibodies (1:200; cat. no. ZX300, Nuoyang, Beijing, China) for $1 \mathrm{~h}$ at room temperature. Subsequently, the sections were washed again with PBS and incubated with DAB reagent (ViGene Biosciences, Inc., China). Finally, images of the stained samples were captured using an optical microscope (Olympus, Tokyo, Japan).

Dual luciferase reporter assay. Genomic DNA was extracted from the endothelial cells using a Genome DNA Extract kit (Kangwei, China). The 2,000, 1,500, 1,000 and $500 \mathrm{bp}$ upstream regions from the transcription initiation site of the ADGRE5 promoter were amplified, gel-purified and sub-cloned into a pGL3-basic luciferase reporter vector (Promega Corporation, USA) between the HindIII and SacI sites. The primer sequences used were as follows: 2 k-promoter, forward 5'-CAAGTCACGCCGAATCCAATA-3' and reverse 5'-CGGTCCTGAACT TTCCGAGATG-3'; $1.5 \mathrm{k}$-promoter, forward 5'-GACGGCTCAGGACCTACA TAA-3' and reverse 5'-CGGTCCTGAACTTTCCGAGAT G-3'; 1k-promoter, forward 5'-GAATCCCAATACGTCAAG CCA-3' and reverse 5'-CGGTCCTGAACTTTCCGAGAT G-3'; 0.5k-promoter, forward 5'-CTAAGCAACCGTGTC GAACAC-3' and reverse 5'-CGGTCCTGAACTTTCCGA GATG-3'. Each vector was transfected into the endothelial cells with glucose treatment (control, $5.5 \mathrm{mM}$; high, $33 \mathrm{mM}$ ) for $24 \mathrm{~h}$. The activity of each was determined using a dual luciferase reporter assay system.

Transcription factor (TF) filter plate assay. TF filter plate (Signosis, Santa Clara, CA, USA) assays were performed according to the manufacturer's protocol. In brief, endothelial cells were collected to extract nuclear proteins. TF DNA complexes were created by mixing TF probes with a $500 \mathrm{bp}$ sequence of the ADGRE5 promoter. Subsequently, complexes were separated from free probes and the bound probes were eluted. Following hybridization of the eluted probes on a hybridization plate, which included nuclear proteins, the relative activity was detected using a luminometer (BioTek Instruments, Inc., Winooski, VT, USA).

Statistical analysis. All significant differences between the mean were analyzed using GraphPad Prism 5.0 software (GraphPad Software, Inc., La Jolla, CA, USA). Data are presented as the mean \pm standard deviation. Comparisons were performed using Student's t-test. $\mathrm{P}<0.05$ was considered to indicate a statistically significant difference.

\section{Results}

Expression of CD97/ADGRE5 in high glucose-induced HUVECs and diabetic mice. The expression of CD97 in endothelial cells subjected to glucose treatment was assessed using western blot analysis and flow cytometry; a basal concentration $(5.5 \mathrm{mM})$ of glucose was used as a control. As shown in Fig. 1A and B, the expression of CD97 was reduced when exposed to an increasing glucose concentration gradient. Alterations in the three CD97 isoforms, CD97 (EGF1,2,5), CD97 (EGF1,2,3,5) and CD97 (EGF1,2,3,4,5), were also analyzed in high glucose-induced endothelial cells. As shown in Fig. 1C, the endothelial cells predominantly expressed CD97 (EGF1,2,5), which was 600 bp in length, and its pattern was altered in response to high glucose treatment. Furthermore, staining of the aortic endothelial tissues from the diabetic mice using anti-CD97 antibody showed lower expression of CD97, compared with the physiological saline-treated group (Fig. 1D).

Overexpression of CD97 (EGF1,2,5) in HUVECs attenuates high glucose-induced dysregulation of migration. To determine whether CD97 (EGF1,2,5) enhanced the dysregulation of endothelial cell migration induced by high glucose, the present study initially constructed a CD97 (EGF1,2,5)-overexpression endothelial cell line via lentivirus transfection (Fig. 2A). As shown in Fig. 2B, high glucose stimulation reduced the mobility ratio of the endothelial cells, whereas the overexpression of CD97 partially attenuated this. 
A

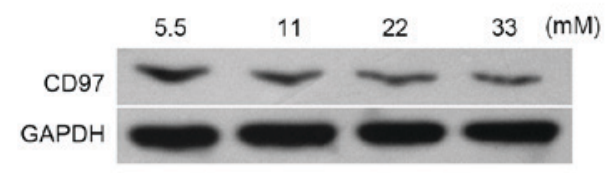

C

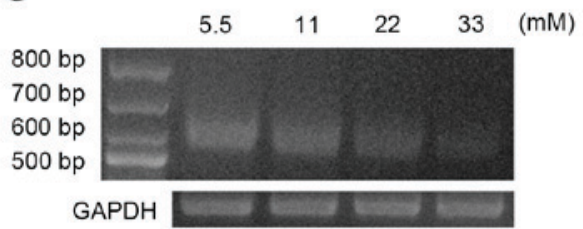

D
B
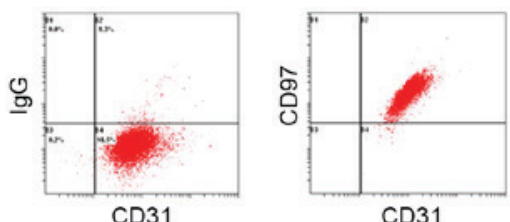

CD31

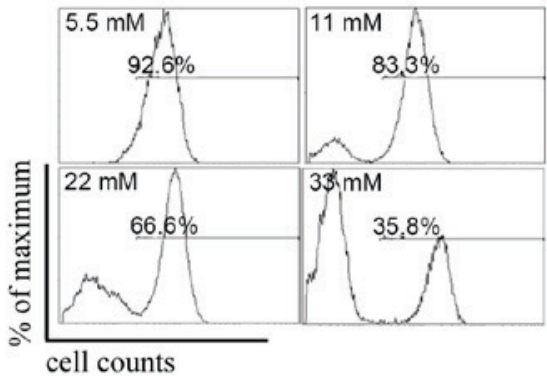

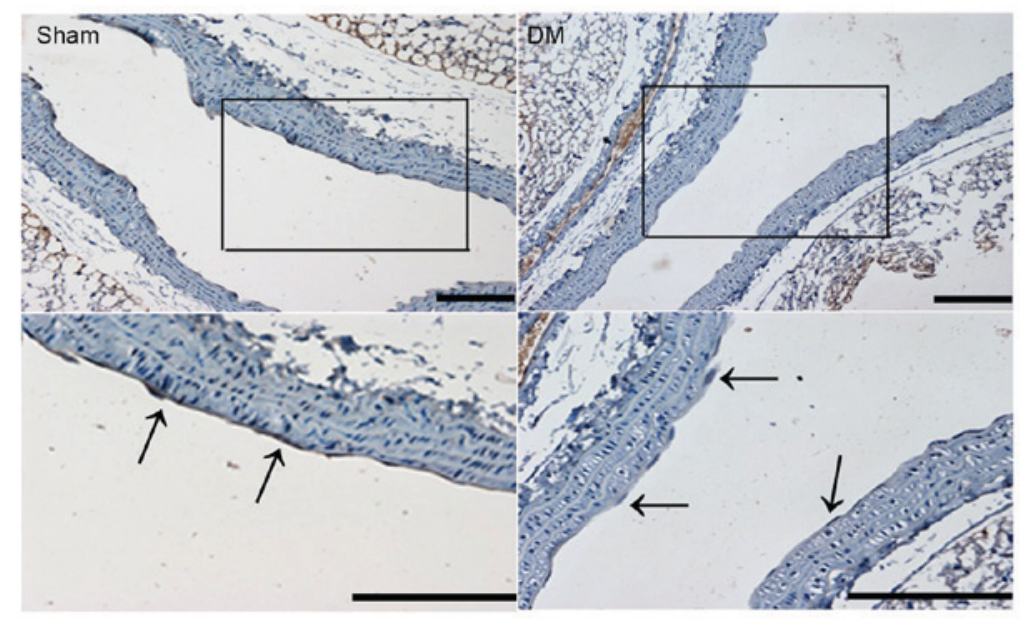

Figure 1. Expression of CD97 is reduced in high glucose-treated endothelial cells and mice with DM. (A) Analysis of the protein expression ofCD97 in response to high glucose (11, 22 and $33 \mathrm{mM}$ ); the basal control condition was $5.5 \mathrm{mM}$ glucose. GAPDH was used as a loading control. (B) Analysis of the expression of CD97 using flow cytometry combined with anti-CD31; IgG was used as a control to treat the endothelial cells (above), or the cells were treated with high glucose $(11,22$ and $33 \mathrm{mM}$ ) with a control of $5.5 \mathrm{mM}$ basal glucose (lower). (C) CD97 mRNA transcript levels were assessed using reverse transcription-polymerase chain reaction analysis in cells treated under conditions of high glucose $(11,22$ and $33 \mathrm{mM})$ and using a basal control of $5.5 \mathrm{mM}$ glucose. Subsequently, CD97 isoforms were separated using agarose gel electrophoresis. GAPDH was used as a loading control. (D) Aortic endothelium samples from mice with DM and healthy control mice were stained using anti-CD97 antibody. Endothelial cells are indicated by black arrows. DM, diabetes mellitus. CD97, cluster of differentiation 97. Scale bar, $60 \mu \mathrm{m}$.

A

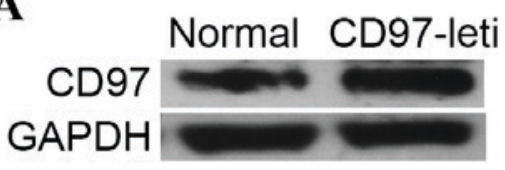

B
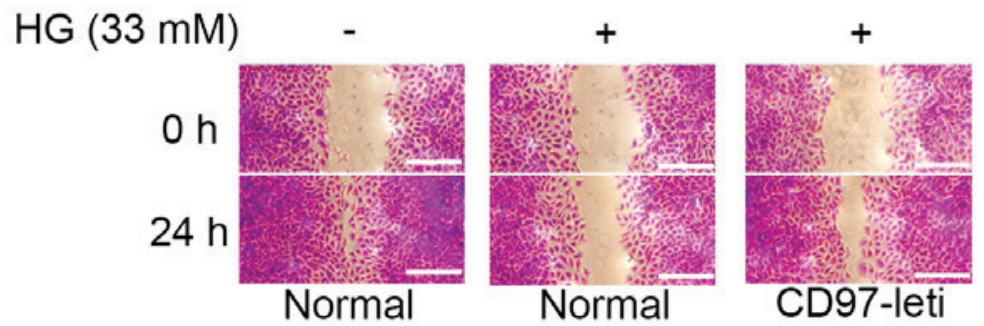

Figure 2. Overexpression of CD97 reverses the dysregulation of high glucose-induced endothelial cell migration. (A) Confirmation of the overexpression of CD97 in endothelial cells transfected with a lentivirus using western blot analysis. (B) Representative images from wound healing assays prior to $(0 \mathrm{~h})$ and following $(24 \mathrm{~h})$ high glucose $(33 \mathrm{mM})$ or basal glucose $(5.5 \mathrm{mM}$; control) treatment of normal and CD97-lentivirus endothelial cells. Scale bar, $70 \mu \mathrm{m}$. CD97, cluster of differentiation 97; HG, high glucose; leti, lentivirus; HG, high glucose. 
A

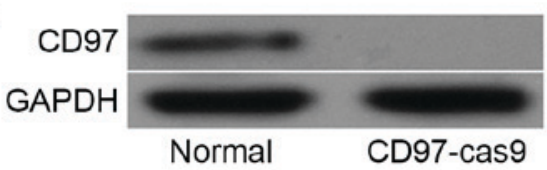

B

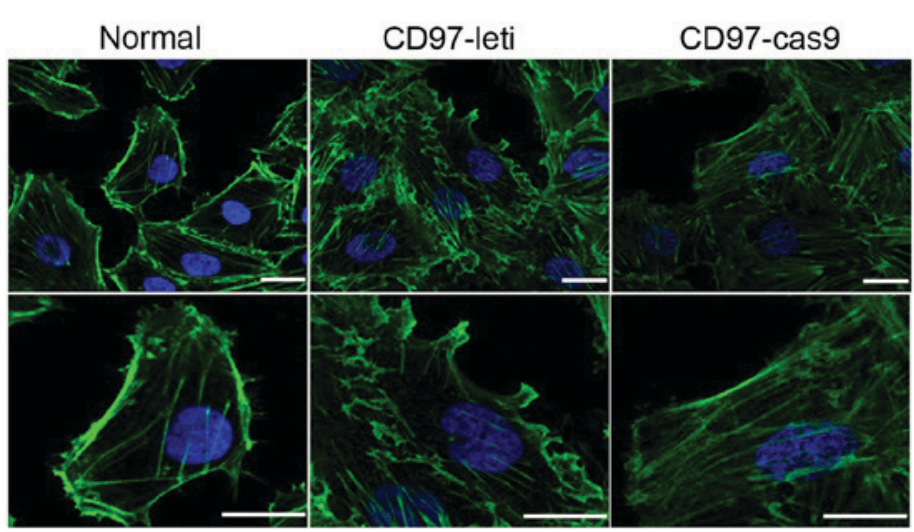

C

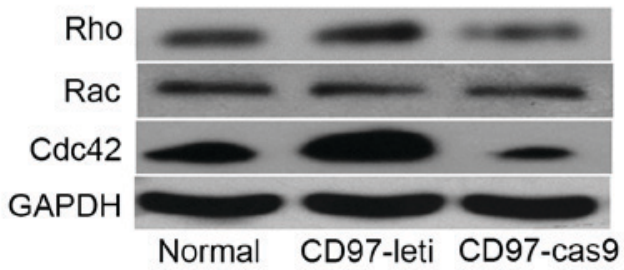

D

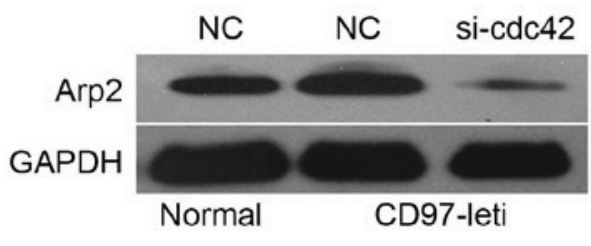

Figure 3. CD97 promotes membrane ruffling and lamellipodia formation in endothelial cells. (A) Validation of the CD97 knockout status of endothelial cells generated by clustered regularly interspaced short palindromic repeats/Cas9 using western blotting. (B) Alterations in the distribution of F-actin in endothelial cells, CD97-Cas9 endothelial cells or CD97-lentivirus endothelial cells. Stress fibers or lamellipodia are indicated by white arrows. Scale bar, $3 \mu \mathrm{m}$. (C) Protein levels of RHO, RAC and CDC42 in endothelial cells, CD97-Cas9 endothelial cells or CD97-lentivirus endothelial cells. (D) Analysis of protein expression levels ofCD97 in endothelial cells, CD97-lentivirus endothelial cells or CD97-lentivirus endothelial cells transfected with siRNA to knockdown the expression of CDC42. CD97, cluster of differentiation 97; Cas9, clustered regularly interspaced short palindromic repeats-associated protein9; Rho, Ras homolog; Rac, Ras-related C3 botulinum toxin substrate; Cdc42, cell division cycle 42; Arp2, actin-related protein 2; NC, negative control; si, small interfering RNA; leti, lentivirus.

CD97 promotes actin enrichment and reorganization in a CDC42-ARP2 dependent manner. To analyze the effects of CD97 in the cytoskeleton of endothelial cells, a CD97-knockout endothelial cell line (CD97-Cas9) was constructed using clustered regularly interspaced short palindromic repeats (CRISPR)/CRISPR-associated protein 9 (Cas9) technology (Fig. 3A). Cytoskeletal staining revealed that the expression level ofCD97 was associated with membrane ruffling and lamellipodia formation (Fig. 3B). In the previous study by Wojciak-Stothard et al (22), RHO, RAC and CDC42 were found to control reorganization of the actin cytoskeleton and to promote migration in endothelial cells. The present study hypothesized that these GTP-binding proteins are also activated in the CD97-overexpressing cell line. As shown in Fig. 3C, the expression of CDC42 was positively regulated by CD97. Furthermore, to elucidate the mechanism underlying the effects of CD97 on cytoskeletal alterations, an evolutionarily conserved actin nucleation factor, the ARP2/3 complex, which is necessary for lamellipodia extension and cell migration in fibroblasts, was examined (23). As shown in Fig. 3D, the upregulation of CD97 increased the levels of ARP2, whereas the downregulation of CDC42, induced by using siRNA to target the mRNA transcripts encoding CDC42, abrogated the increase levels of ARP2. This suggested that CD97 promoted lamellipodia formation, which was dependent upon the activation of CDC42 and ARP2.

High glucose inhibits CD97 transcription via the regulation of STAT1. The mechanism underlying the regulatory effect of high glucose on the expression of CD97 was also examined in detail. To characterize the promoter region of CD97, a series of luciferase reporter plasmids, including the 500, 1,000, 1,500 and $2,000 \mathrm{bp}$ sequences upstream of the transcription start point (24), were constructed (Fig. 4A). Dual luciferase reporter assays revealed that the promoters, which included a $500 \mathrm{bp}$ sequence, represented the minimal length required to suppress CD97 transcription. Subsequently, TF filter plate assays were performed using this 500 bp sequence of the CD97 promoter. As shown in Fig. 4B, STAT1 was the most prominent factor to be activated by high glucose concentrations; this factor also bound to the $500 \mathrm{bp}$ promoter region upstream of CD97. Therefore, the present study aimed to characterize the role of STAT1 in the regulation of CD97 transcription. Nuclear extracts from high glucose-induced endothelial cells showed higher expression levels of STAT1 (Fig. 4C). Additionally, 
A

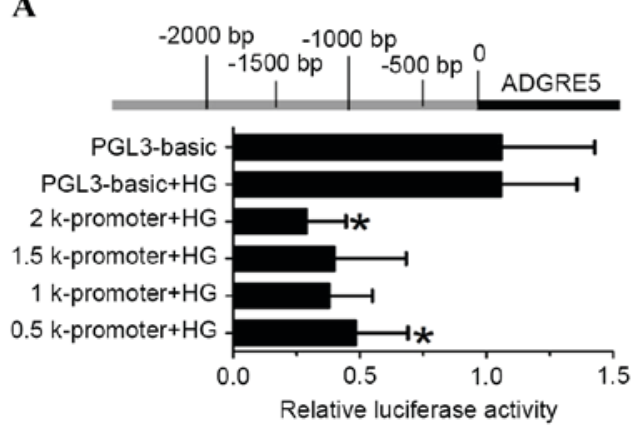

C

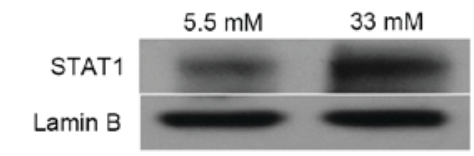

E

CD97 promoter

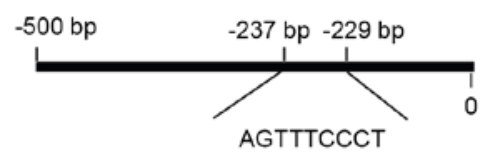

B

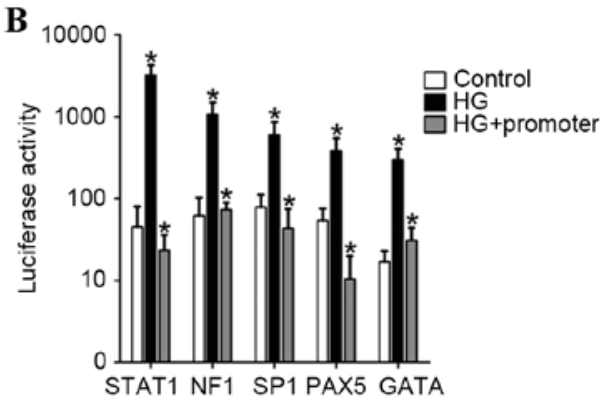

D
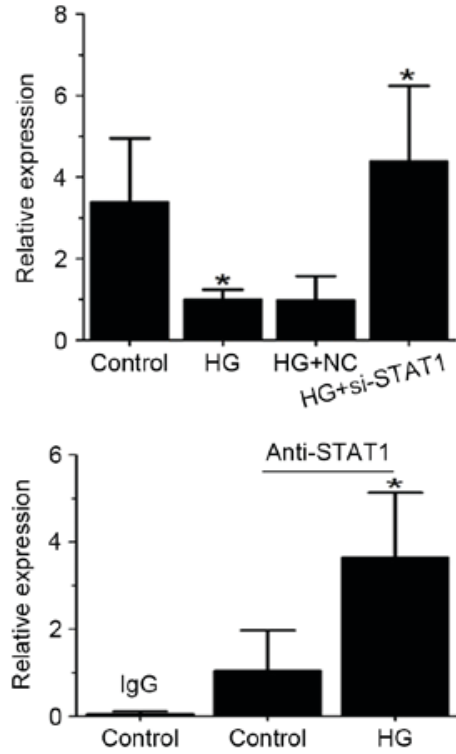

Figure 4. High glucose concentrations inhibit CD97 transcription via the upregulation of STAT1. (A) Schematic representation of the promoter regions (upper), which were sub-cloned into the pGL3-basic luciferase reporter. Activation of the promoter-luciferase reporters in response to high glucose concentrations in endothelial cells is shown below. (B) Top five most robust binding transcription factors in the CD97 promoter region, determined using TF filter plate assays. (C) High glucose $(33 \mathrm{mM})$ stimulation promoted STAT1 nuclear translocation; $5.5 \mathrm{mM}$ glucose stimulation was used as a control. (D) Analysis of the mRNA transcript expression of CD97 in endothelial cells transfected with siRNA targeting STAT1 prior to high glucose ( $33 \mathrm{mM}$ ) treatment (5.5 mM glucose served as a control). (E) Schematic illustration of potential binding sites in the CD97 promoter for STAT1. High glucose (33 mM) treatment increased STAT1 binding to its binding site in the CD97 promoter (5.5 mM glucose served as a control). ${ }^{*} \mathrm{P}<0.05$. CD97, cluster of differentiation 97; ADGRE5, adhesion G protein-coupled receptor 5; STAT1, signal transducer and activator of transcription 1; NF1, neurofibromatosis type 1; SP1, specificity protein 1; PAX5, paired box 5; GATA, GATA binding protein; $\mathrm{HG}$, high glucose; si, small interfering RNA.

transfection with siRNA targeting STAT1 under high glucose conditions revealed that high glucose resulted in reduced expression levels of CD97 via the upregulation of STAT1 (Fig. 4D). The effect of high glucose on the binding activity of STAT1 to the CD97 promoter was also examined using ChIP assays. As shown in Fig. 4E, high levels of glucose increased the binding activity of STAT1 at the CD97 promoter.

\section{Discussion}

CD97/ADGRE5 belongs to the GPCR family (11-13) and has been found to regulate migration in multiple cell types, including granulocytes (25), prostate cancer cells (19) and endothelial cells (9). In the present study, the role of CD97 in high glucose-induced dysregulation of endothelial cell migration was reported. By activating CDC42 and ARP2, CD97 promotes the formation and extension of lamellipodia by endothelial cells. Unlike prostate cancer cells, for which CD97-mediated invasion is primarily RHO-dependent (19), CD97 primarily regulates the expression of CDC42 rather than RHO in endothelial cells. This difference may be attributed to differences in cell type or treatment. In addition, the present study found that specificity protein 1 (SP1) controlled the transcription of CD97 in smooth muscle cells (26) and regulated the transcription of CD97 under conditions of high glucose treatment. However, compared with the STAT1 transcription factor, SP1 exhibits lower activity in high glucose-stimulated endothelial cell assays. However, in endothelial cells stimulated by high glucose concentrations, whether these two factors can promote the transcription of CD97 in a cooperative manner remains to be elucidated. Additionally, whether CD97 acts in high glucose-induced apoptosis or other modes of dysfunction in endothelial cells remains to be elucidated. The present study is the first, to the best of our knowledge, to describe a link between CD97 and the dysregulation of high glucose-induced endothelial migration, which may provide insights in the identification of novel therapeutic targets for the treatment of diabetic complications.

In conclusion, the overexpression of CD97 reversed the dysregulation of high glucose-induced endothelial cell migration by activating $\mathrm{CDC} 42$, which acts via its downstream signaling adaptor ARP2. 


\section{References}

1. Creager MA, Lüscher TF, Cosentino F and Beckman JA: Diabetes and vascular disease: Pathophysiology, clinical consequences, and medical therapy: Part I, Circulation 108: 1527-1532, 2003.

2. Antonetti DA, Barber AJ, Khin S, Lieth E, Tarbell JM and Gardner TW: Vascular permeability in experimental diabetes is associated with reduced endothelial occludin content: Vascular endothelial growth factor decreases occludin in retinal endothelial cells. Penn State Retina Research Group, Diabetes 47 1953-1959, 1998

3. Campanini M, Airoldi G, Cusinato S, Ballarè $M$ and Monteverde A: Arterial blood pressure as a factor in endothelial permeability. J Hypertens Supp1 9: S200-S201, 1991.

4. Bassenge $\mathrm{E}$ : Clinical relevance of endothelium-derived relaxing factor (EDRF). Br J Clin Pharmacol 34 (Suppl 1): S37-S42, 1992.

5. Cohen RA: The role of nitric oxide and other endothelium-derived vasoactive substances in vascular disease, Prog Cardiovasc Dis 38: 105-128, 1995.

6. De Meyer GR and Herman AG: Vascular endothelial dysfunction. Prog Cardiovasc Dis 39: 325-342, 1997.

7. Kario K, Matsuo T, Kobayashi H, Matsuo M, Sakata T and Miyata T: Activation of tissue factor-induced coagulation and endothelial cell dysfunction in non-insulin-dependent diabetic patients with microalbuminuria, Arterioscler Thromb Vasc Biol 15: 1114-1120, 1995.

8. Hamuro M, Polan J, Natarajan M and Mohan S: High glucose induced nuclear factor kappa B mediated inhibition of endothelial cell migration. Atherosclerosis 162: 277-287, 2002.

9. Wang T, Ward Y, Tian L, Lake R, Guedez L, Stetler-Stevenson WG and Kelly K: CD97, an adhesion receptor on inflammatory cells, stimulates angiogenesis through binding integrin counterreceptors on endothelial cells. Blood 105: 2836-2844, 2005.

10. Hamann J, Hartmann E and van Lier RA: Structure of the human CD97 gene: Exon shuffling has generated a new type of seven-span transmembrane molecule related to the secretin receptor superfamily. Genomics 32: 144-147, 1996.

11. Kwakkenbos MJ, Kop EN, Stacey M, Matmati M, Gordon S, Lin HH and Hamann J: The EGF-TM7 family: A postgenomic view. Immunogenetics 55: 655-666, 2004.

12. Leemans JC, te Velde AA, Florquin S, Bennink RJ, de Bruin K, van Lier RA, van der Poll T and Hamann J: The epidermal growth factor-seven transmembrane (EGF-TM7) receptor CD97 is required for neutrophil migration and host defense. J Immunol 172: 1125-1131, 2004.

13. McKnight AJ and Gordon S: EGF-TM7: A novel subfamily of seven-transmembrane-region leukocyte cell-surface molecules. Immunol Today 17: 283-287, 1996.
14. Aust G, Eichler W, Laue S, Lehmann I, Heldin NE, Lotz O, Scherbaum WA, Dralle H and Hoang-Vu C: CD97: A dedifferentiation marker in human thyroid carcinomas. Cancer Res 57: 1798-1806, 1997.

15. Eichler W, Aust G and Hamann D: Characterization of an early activation-dependent antigen on lymphocytes defined by the monoclonal antibody BL-Ac (F2). Scand J Immunol 39: 111-115, 1994.

16. Hamann J, Eichler W, Hamann D, Kerstens HM, Poddighe PJ, Hoovers JM, Hartmann E, Strauss M and van Lier RA: Expression cloning and chromosomal mapping of the leukocyte activation antigen CD97, a new seven-span transmembrane molecule of the secretion receptor superfamily with an unusual extracellular domain. J Immunol 155: 1942-1950, 1995.

17. Jaspars LH, Vos W, Aust G, Van Lier RA and Hamann J: Tissue distribution of the human CD97 EGF-TM7 receptor. Tissue Antigens 57: 325-331, 2001

18. Steinert M, Wobus M, Boltze C, Schütz A, Wahlbuhl M, Hamann $\mathrm{J}$ and Aust G: Expression and regulation of CD97 in colorectal carcinoma cell lines and tumor tissues. Am J Pathol 161: 1657-1667, 2002.

19. Ward Y, Lake R, Yin JJ, Heger CD, Raffeld M, Goldsmith PK, Merino $M$ and Kelly K: LPA receptor heterodimerizes with CD97 to amplify LPA-initiated RHO-dependent signaling and invasion in prostate cancer cells. Cancer Res 71: 7301-7311, 2011.

20. Livak KJ and Schmittgen TD: Analysis of relative gene expression data using real-time quantitative PCR and the 2(-Delta Delta $\mathrm{C}(\mathrm{T})$ ) method. Methods 25: 402-408, 2001.

21. Ran FA, Hsu PD, Wright J, Agarwala V, Scott DA and Zhang F: Genome engineering using the CRISPR-Cas9 system. Nat Protoc 8: 2281-2308, 2013.

22. Wójciak-Stothard B, Entwistle A, Garg R and Ridley AJ: Regulation of TNF-alpha induced reorganization of the actin cytoskeleton and cell-cell junctions by Rho, Rac, and Cdc42 in human endothelial cells. J Cell Physiol 176: 150-165, 1998.

23. Suraneni P, Rubinstein B, Unruh JR, Durnin M, Hanein D and Li R: The Arp2/3 complex is required for lamellipodia extension and directional fibroblast cell migration. J Cell Biol 197: 239-251, 2012.

24. Li P, Grgurevic S, Liu Z, Harris D, Rozovski U, Calin GA, Keating MJ and Estrov Z: Signal transducer and activator of transcription-3 induces microRNA-155 expression in chronic lymphocytic leukemia. PLoS One 8: e64678, 2013.

25. Veninga H, Becker S, Hoek RM, Wobus M, Wandel E, van der Kaa J, van der Valk M, de Vos AF, Haase H, Owens B, et al: Analysis of CD97 expression and manipulation: Antibody treatment but not gene targeting curtails granulocyte migration. J Immunol 181: 6574-6583, 2008.

26. Wobus M, Wandel E, Prohaska S, Findeiss S, Tschöp K and Aust G: Transcriptional regulation of the human CD97 promoter by Sp1/Sp3 in smooth muscle cells. Gene 413: 67-75, 2008. 